\title{
ON THE SET OF SOLUTIONS OF A CLASS OF NONLINEAR EVOLUTION INCLUSIONS
}

\author{
By Nikolaos S. PapageORgiou $* * * *$
}

\begin{abstract}
In this paper first we establish the existence of solutions for a large class of nonlinear, nonconvex evolution inclusions and then we show that the solution multifunction has a continuous selector. Then we use that selector result to establish the existence of periodic trajectories and we show how this result can be used in nonlinear closed loop (feedback) control systems.
\end{abstract}

\section{Introduction.}

In a recent paper Cellina [8], proved the existence of a continuous selection for the multifunction $S(\xi)$, which to each initial point $\xi$ of a differential inclusion in $\boldsymbol{R}^{n}$ with Lipschitz continuous orientor field associates the set of all trajectories emanating from that point. His result was extended to differential inclusions in a separable Banach space by Colombo-Fryszkowski-RzezuchowskiStaicu [9] and very recently by Staicu [20] to evolution inclusions driven by a time invariant maximal monotone operator defined on a separable Hilbert space. Evolution inclusions are important in the study of infinite dimensional control systems (see Ahmed [1], Avgerinos-Papageorgiou [4] and Papageorgiou [19]). Staicu's proof [20], followed that of [9] which in turn was based on Filippov's approach to the relaxation problem [11].

In this paper we extend the work of Staicu [20] to a larger class of nonlinear evolution inclusions, with a time varying monotone multivalued operator. First we establish the existence of a solution for the multivalued Cauchy problem under consideration. Then following the approach of Staicu, we prove the existence of a continuous solution selector. Having this selector and using a viability result of Avgerinos-Papageorgiou [3], we establish the existence of a periodic solution for an autonomous evolution inclusion. This result extends

* Research supported by NSF Grant DMS-8802688.

** 1980 AMS Subject Classification: 34A60.

Key words and phrases: Multivalued operator, monotone operator, measurable multifunction, upper and lower semicontinuous multifunctions, evolution triple, compact embedding, Shauder-Tichonov fixed point theorem, periodic trajectories.

Received August 2, 1991 ; revised April 7, 1992. 
proposition 4.1 of Staicu [20], which was proved for differential inclusions in a separable Banach space, with no monotone operator present.

\section{Preliminaries.}

Let $(\Omega, \Sigma)$ be a measurable space and $X$ a separable Banach space. By $P_{f(c)}(X)$ we will be denoting the family of nonempty, closed (and convex) subsets of $X$. A multifunction $F: \Omega \rightarrow P_{f}(X)$ is said to be measurable if and only if for every $z \in X, \omega \rightarrow d(z, F(\omega))=\inf \{\|z-x\|: x \in F(\omega)\}$ is measurable. A multifunction $G: \Omega \rightarrow 2^{X} \backslash\{\emptyset\}$ is said to be graph measurable if and only if $G r G=$ $\{(\omega, x) \in \Omega \times X: x \in F(\omega)\} \in \Sigma \times B(X)$ with $B(X)$ being the Borel $\boldsymbol{\sigma}$-field of $X$. For $P_{f}(X)$-valued multifunctions, measurability implies graph measurability and the converse is true provided there exists a $\sigma$-finite measure $\mu(\cdot)$ on $\Sigma$ with respect to which $\Sigma$ is complete. If we have such a measure on $\Sigma$, by $S_{F}^{p} 1 \leqq p \leqq \infty$, we will denote the set of all selectors of $F(\cdot)$ that belong in the LebesgueBochner space $L^{p}(X)$; i.e. $S_{F}^{p}=\left\{f \in L^{p}(X): f(\omega) \in F(\boldsymbol{\omega}) \mu\right.$-a.e. $\}$. This set may be empty. For a measurable multifunction $F(\cdot)$ it is nonempty if and only if $\omega \rightarrow$ $\{\|x\|: x \in F(\omega)\} \in L_{+}^{p}$. For further details on measurable multifunctions we refer to Wagner [21].

A map $A: X \rightarrow 2^{X *}$ is said to be monotone if and only if for all $\left[x, x^{*}\right]$, $\left[y, y^{*}\right] \in G r A$, we have $\left\langle x^{*}-y^{*}, x-y\right\rangle \geqq 0$, where by $\langle\cdot, \cdot\rangle$ we denote the duality brackets of the pair $\left(X, X^{*}\right)$. We say that $A(\cdot)$ is maximal monotone if and only if it is monotone and its graph is not properly included in the graph of another monotone operator. For a maximal monotone operator $A: X \rightarrow 2^{X *}$, we know that for every $x \in X A(x)$ is a convex and $w^{*}$-closed set (possibly empty) and $G r A$ is demiclosed (i.e. closed in $X \times X_{w^{*}}^{*}$ denotes the Banach space $X^{*}$ endowed with the $w^{*}$-topology).

A mapping $J: X \rightarrow 2^{X *}$ is called a duality mapping if it satisfies the following property:

$$
J(x)=\left\{x^{*} \in X^{*}=\left\langle x^{*}, x\right\rangle=\|x\|^{2}=\left\|x^{*}\right\|^{2}\right\} .
$$

If $X$ is a Hilbert space, $J(\cdot)$ reduces to the canonical isomorphism between $X$ and $X^{*}$. In general note that the Hahn-Banach theorem guarantees that for every $x \in X, J(x)$ is a nonempty, closed and convex subset of $X^{*}$. Furthermore if $X^{*}$ is strictly convex, then $J(\cdot)$ is single-valued, surjective, demicontinuous (i.e. if $x_{n} \stackrel{s}{\rightarrow} x$ in $X$ then $J\left(x_{n}\right) \stackrel{w}{\rightarrow} J(x)$ in $X^{*}$ ), maximal monotone, bounded (i.e. maps bounded sets into bounded sets) and coercive (in fact from the definition we have $\langle J(x), x\rangle=\|x\|^{2}$, so $\lim _{\|x\| \rightarrow \infty} \frac{\langle J(x), x\rangle}{\|x\|}=\lim _{\|x\| \rightarrow \infty}\|x\|=+\infty$, coercivity). Another way to define a duality map is to let $\phi: X \rightarrow \boldsymbol{R}_{+}$be defined by $\phi(x)=\|x\|^{2} / 2$ and set $J(x)=\partial \phi(x)$, where $\partial \phi(\cdot)$ denotes the convex subdifferential of $\phi(\cdot)$. For further details on monotone operators and duality maps we refer to Zeidler [22].

Let $Y, Z$ be Hausdorff topological spaces and let $G: Y \rightarrow 2^{Z} \backslash\{\emptyset\}$ be a mul- 
tifunction. We say that $G(\cdot)$ is upper semicontinuous (u.s.c.) (resp. lower semicontinuous (1.s.c.)) if and only if for all $C \cong Z$ closed $G^{-}(C)=\{y \in Y: G(y) \cap C \neq \emptyset\}$ (resp. $G^{+}(C)=\{y \in Y: G(y) \subseteq C\}$ ) is closed in $Y$. If $Y, Z$ are metric spaces, then lower semicontinuity is equivalent to saying that: " $y_{n} \rightarrow y$ in $Y$ implies $G(y) \subseteq$ $\underline{\lim } G\left(y_{n}\right)=\left\{z \in X: z=\lim z_{n}, z_{n} \in G\left(y_{n}\right), n \geqq 1\right\}=\left\{z \in X: \lim d_{z}\left(z, G\left(y_{n}\right)\right)=0\right\}$, with $d_{Z}(\cdot, \cdot)$ being the metric on $Z$. Also if $Z$ is a metric space, on $P_{f}(Z)$ we can define a generalized metric known in the literature as Hausdorff metric by setting

$$
h(A, B)=\max \left[\sup _{a \in A} d(a, B), \sup _{b \in B} d(b, A)\right]
$$

for all $A, B \in P_{f}(Z)$. If $Z$ is complete, then so is $\left(P_{f}(Z), h\right)$.

\section{Continuous selector.}

The mathematical setting of our problem is the following. Let $T=[0, b]$, $H$ a separable Hilbert space and $X$ a dense subspace of $H$, carrying the structure of a separable reflexive Banach space which embeds continuously into $X$. Identifying $H$ with its dual (pivot space), we have $X \hookrightarrow_{\rightarrow} H \subseteq X^{*}$, with all embeddings being continuous and dense. Such a triple of spaces is known in the literature as "evolution triple" or "Gelfand triple" (see Zeidler [22]). We will assume that the embeddings are also compact. By $\langle\cdot, \cdot\rangle$ we will denote the duality brackets for the pair $\left(X, X^{*}\right)$ and by $(\cdot, \cdot)$ the inner product of $H$. The two are compatible in the sense that $\left.\langle\cdot, \cdot\rangle\right|_{X \times H}=(\cdot, \cdot)$. Also by $\|\cdot\|$ (resp. $|\cdot|,\|\cdot\|_{*}$ ), we will denote the norm of $X$ (resp. of $H, X^{*}$ ).

Recall that a map $A: X \rightarrow X^{*}$ is hemicontinuous if and only if the real function $\lambda \rightarrow\langle A(x+\lambda y), v\rangle$ is continuous on $[0,1]$ for all $x, y, v \in X$. It is wellknown (see for example Zeidler [22]), that a monotone, hemicontinuous, everywhere defined operator is maximal monotone. In the next lemma we show that a similar result is also true for multivalued monotone operators. Let $P_{w k c}\left(X^{*}\right)=\left\{A \subseteq X^{*}\right.$ : nonempty, $w$-compact, convex $\}$.

LEMMA 3.1. If $A: X \rightarrow P_{w k c}\left(X^{*}\right)$ is a multivalued operator s.t.

(1) $x \rightarrow A(x)$ is monotone,

(2) $\lambda \rightarrow A(x+\lambda y)$ is u.s.c. from $[0,1]$ into $X_{w}^{*}$ for every $x, y \in X$ (here by $X_{w}^{*}$ we denote the Banach space $X^{*}$ endowed with the weak topology), then $A(\cdot)$ is maximal monotone.

Proof. We need to show that if $\left\langle y^{*}-x^{*}, y-x\right\rangle \geqq 0$ for all $\left[x, x^{*}\right] \in G r A$, then $\left[y, y^{*}\right] \in G r A$. Suppose not. Then $y^{*} \notin A(y)$. Since the latter is $w$-compact and convex, using the strong separation theorem, we can find $v \in X$ s.t.

$$
\sigma(v ; A(y))=\sup \left\{\left\langle z^{*}, v\right\rangle: z^{*} \in A(y)\right\}\left\langle\left\langle y^{*}, v\right\rangle\right.
$$

Let $v_{\lambda}=y+\lambda v \quad \lambda \in[0,1]$ and $v_{\lambda}^{*} \in A\left(v_{\lambda}\right)$. We have 


$$
0 \leqq\left\langle v_{\wedge}^{*}-y^{*}, y-y+\lambda v\right\rangle \Longrightarrow 0 \leqq\left\langle v_{\lambda}^{*}-y^{*}, v\right\rangle \quad \text { for every } \lambda \in[0,1] \text {. }
$$

Note that since $X_{w}^{*}$ is a regular topological space and by hypothesis (2) the multifunction $\alpha: \lambda \rightarrow A(y+\lambda v)$ is u.s.c. from $[0,1]$ into $X_{w}^{*}$, it has a closed graph in $[0,1] \times X_{w}^{*}$ (see Klein-Thompson [15]). Furthermore, since by hypothesis (1), $A(\cdot)$ is monotone and is defined everywhere, is locally bounded (see Zeidler [22], proposition 32.33 , p. 884). So for $\lambda \in[0,1]$ small enough, the set $\left\{v_{\lambda}^{*}\right\}$ is bounded. So if $\lambda_{n} \downarrow 0, v_{\lambda_{n}} \stackrel{s}{\rightarrow} y$ in $X$ and by passing to a subsequence if necessary, we may assume that $v_{\lambda_{n}}^{*} \stackrel{w}{\rightarrow} v^{*}$ in $X^{*}$. Note that $\left[\lambda_{n}, v_{\lambda_{n}}^{*}\right] \in G r \alpha \Rightarrow\left[0, v^{*}\right] \in G r \alpha \Rightarrow\left[y, v^{*}\right] \in$ GrA. Also in the limit we get

$$
0 \leqq\left\langle v^{*}-y^{*}, v\right\rangle \Longrightarrow\left\langle y^{*}, v\right\rangle \leqq\left\langle v^{*}, v\right\rangle \leqq \sigma(v ; A(y))
$$

Comparing (1) and (2) above we get the desired contradiction. Thus indeed $\left[y, y^{*}\right] \in G r A$, which proves the maximal monotonicity of the multivalued operator $A(\cdot)$.

Q.E.D.

Next we prove a superpositional measurability result for time-varying maximal monotone multivalued operators. Additional results on the joint and superpositional measurability of multifunctions can be found in [18].

LEMMA 3.2. If $A: T \times X \rightarrow 2^{X^{*}} \backslash\{\emptyset\}$ is a multivalued operator s.t.

(1) $(t, x) \rightarrow A(t, x)$ is graph measurable,

(2) $x \rightarrow A(t, x)$ is maximal monotone,

then for every $x: T \rightarrow X$ measurable, $t \rightarrow A(t, x(t))$ is a measurable multifunction from $T$ with the Lebesgue $\sigma$-field into $P_{f c}\left(X^{*}\right)$.

Remark. Because of the maximal monotonicity hypothesis, for every $(t, x)$ $\in T \times X$ we have $A(t, x) \in P_{f c}\left(X^{*}\right)$ (see section 2). Also note that for every $t \in T$ $\operatorname{Gr} A(t, \cdot)$ is closed in $X \times X_{w}^{*}$ (demiclosed). Hence, since $B\left(X^{*}\right)=$ the Borel $\sigma$ field of $X^{*}$ equals $B\left(X_{w}^{*}\right)=$ the Borel $\sigma$-field of $X_{w}^{*}$ (see Edgar [23], corollary 2.4), we see that hypothesis (1) is equivalent to saying that $t \rightarrow G r A(t, \cdot)$ is measurable from $T$ into $P_{f c}\left(X \times X_{w}^{*}\right)$.

Proof. Let $R: T \rightarrow P_{f c}\left(X^{*}\right)$ be the multifunction defined by

$$
R(t)=\left\{x^{*} \in X^{*}: x^{*} \in A(t, x(t))\right\}
$$

From the remark above we know that hypothesis (1) is equivalent to the measurability of $t \rightarrow G r A(t, \cdot)$. Hence from theorem 4.2 of Wagner [21], we know that we can find $x_{n}: T \rightarrow X, x_{n}^{*}: T \rightarrow X^{*} n \geqq 1$ measurable functions s.t. for all $t \in T$ we have

$$
\operatorname{Gr} A(t, \cdot)=\operatorname{cl}\left\{\left[x_{n}(t), x_{n}^{*}(t)\right]\right\}_{n \geqq 1}
$$

Then since by hypothesis $(2), A(t, \cdot)$ is maximal monotone, we have 


$$
\begin{aligned}
G r R & =\bigcap_{n \geq 1}\left\{\left(t, x^{*}\right) \in T \times X^{*}:\left\langle X^{*}-x_{n}^{*}(t), x(t)-x_{n}(t)\right\rangle \geqq 0\right\} \in B(T) \times B\left(X^{*}\right) \\
& \Longrightarrow t \rightarrow A(t, x(t)) \text { is graph measurable, hence Lebesgue measurable; i.e. }
\end{aligned}
$$

$\left(\mathcal{L}, B\left(X^{*}\right)\right.$ )-measurable, with $\mathcal{L}$ being the Lebesgue $\sigma$-field on $T$ (the Lebesgue completion of $B(T)$ ).

Q.E.D.

Let $W(T)=\left\{x \in L^{2}(X): \dot{x} \in L^{2}\left(X^{*}\right)\right\}$. Here the derivative is understood in the sense of vector valued distributions. When furnished with the norm $\|x\|_{W(T)}=$ $\left(\|x\|_{L^{2}(X)}^{2}+\|\dot{x}\|_{L^{2}\left(X^{*}\right)}^{2}\right)^{1 / 2}, W(T)$ becomes a separable, reflexive Banach space. Furthermore $W(T) \hookrightarrow C(T, H)=$ space of continuous functions from $T$ into $H$ \} continuously; i.e. every function in $W(T)$, after possible modification on a set of measure zero, equals a continuous function from $T$ into $H$. Also since $X \hookrightarrow H$ compactly, then $W(T) \hookrightarrow L^{2}(H)$ compactly (see Zeidler [22], p. 450) and if $X$ is a separable Hilbert space and $X \subseteq H$ compactly, then $W(T) \subseteq C(T, H)$ compactly (see Nagy [16]).

We examine the following multivalued Cauchy problem:

$$
\left\{\begin{array}{l}
\dot{x}(t) \in-A(t, x(t))+f(t) \text { a. e. } \\
x(0)=\xi
\end{array}\right\}(*)^{\prime}
$$

Our hypothesis on the multivalued operator $A(t, x)$ is the following

$H(A): \quad A: T \times X \rightarrow 2^{X *} \backslash\{\emptyset\}$ is a multivalued operator s.t.

(1) $(t, x) \rightarrow A(t, x)$ is graph measurable,

(2) $\lambda \rightarrow A(t, x+\lambda y)$ is u.s.c. from $[0,1]$ into $X_{w}^{*}$,

(3) $x \rightarrow A(t, x)$ is monotone,

(4) $c\|x\|^{2} \leqq r(x ; A(t, x))=\inf \left\{\left\langle v^{*}, x\right\rangle: v^{*} \in A(t, x)\right\}$ a. e. with $c>0$,

(5) $|A(t, x)|=\sup \left\{\left\|v^{*}\right\|_{*}: v^{*} \in A(t, x)\right\} \leqq a(t)+b^{\prime}\|x\|$ a. e. with $a(\cdot) \in L_{+}^{2}, b^{\prime} \geqq 0$.

LEMmA 3.3. If hypothesis $H(A)$ holds, $f \in L^{2}(H)$ and $\xi \in H$, then $(*)^{\prime}$ admits a unique solution $x(\cdot) \in W(T)$.

Proof. Let $E: D(E) \subseteq L^{2}(X) \rightarrow L^{2}\left(X^{*}\right)$ be the operator defined by

$$
(E x)(\cdot)=\frac{d x}{d t}(\cdot)
$$

with the time derivative defined in the sense of vector-valued distributions on $(0, b)$ and $D(E)=\left\{x \in L^{2}(X): \dot{x} \in L^{2}\left(X^{*}\right), x(0)=\xi\right\} \subseteq W(T)$. We claim that $E$ is maximal monotone. By renorming $X, X^{*}$ if necessary, we may assume that both spaces are locally uniformly convex (in particular then strictly convex). This can be done by Troyanski's theorem; see Zeidler [22], p. 862. Denote by $((\cdot, \cdot))_{0}$ the duality brackets for the pair $\left(L^{2}(X), L^{2}\left(X^{*}\right)\right)$. We have for all $x, y$ $\in D(E)$ 


$$
((E(x)-E(y), x-y))_{0}=\int_{0}^{b}\langle\dot{x}(t)-\dot{y}(t), x(t)-y(t)\rangle d t=\frac{1}{2}|x(b)-y(b)|^{2} \geqq 0 .
$$

(see Zeidler [22], proposition 23.23 (iv), p. 423). So $E(\cdot)$ is monotone. Hence to establish its maximal monotonicity, we need to show that Range $(E+\hat{J})=$ $L^{2}\left(X^{*}\right)$ where $\hat{J}: L^{2}(X) \rightarrow L^{2}\left(X^{*}\right)$ is the duality map. Note that $\hat{J}(x)(\cdot)=J(x(\cdot))$, where $J$ is the duality map from $X$ into $X^{*}$, and we know (see section 2), that $J(\cdot)$ is monotone, hemicontinuous and $\langle J(x), x\rangle=\|x\|^{2}$. Let $h \in L^{2}\left(X^{*}\right)$. Then there exists $x \in D(E)$ s.t. $E(x)+\hat{J}(x)=h$ if and only if the evolution equation $\dot{x}(t)+J(x(t))=h(t)$ a.e., $x(0)=\xi$ has a solution. Since $J(\cdot)$ is monotone, demicontinuous bounded, by theorem 30.A of Zeidler [22], the equation has a unique solution $x(\cdot) \in W(T)$. Since $h \in L^{2}\left(X^{*}\right)$ was arbitrary, we conclude that Range $(E+\hat{J})=L^{2}\left(X^{*}\right) \Rightarrow E$ is indeed maximal monotone.

Next let $\hat{A}(x)=S_{A(\cdot, x(\cdot))}^{2}$ (the realization of $A(t, \cdot)$ on $\left.L^{2}\left(X^{*}\right)\right)$. Clearly because of the monotonicity of $A(t, \cdot), \hat{A}: L^{2}(X) \rightarrow P_{w k c}\left(L^{2}\left(X^{*}\right)\right)$ is monotone too. Also if $x, y \in L^{2}(X)$ and $\hat{\alpha}:[0,1] \rightarrow P_{w k c}\left(L^{2}\left(X^{*}\right)\right)$ is defined by $\hat{\alpha}(\lambda)=\hat{A}(x+\lambda y)$, then we claim that $\hat{\alpha}(\cdot)$ is u.s.c. from $[0,1]$ into $L^{2}\left(X^{*}\right)_{w}$. To this end we need to show that given $C$ a weakly closed subset of $L^{2}\left(X^{*}\right)$ we have that $\hat{\alpha}^{-}(C)=\{\lambda \in[0,1]: \hat{\alpha}(\lambda) \cap C \neq \emptyset\}$ is closed in $[0,1]$. So let $\lambda_{n} \in \hat{\alpha}^{-}(C) n \geqq 1 \lambda_{n} \rightarrow \lambda$ in $[0,1]$. Let $f_{n} \in \hat{\alpha}\left(\lambda_{n}\right) \cap C$. Because of hypothesis $H(A)(5),\left\{f_{n}\right\}_{n \geq 1} \subseteq L^{2}\left(X^{*}\right)$ is bounded. So by passing to a subsequence if necessary we may assume that $f_{n} \stackrel{w}{\rightarrow} f$ in $L^{2}\left(X^{*}\right)$. Then from theorem 3.1 of [17] we have

$$
\begin{aligned}
f(t) & \in \overline{\operatorname{conv}} w-\varlimsup \overline{\lim }\left\{f_{n}(t)\right\}_{n \geqq 1} \\
& \cong \overline{\operatorname{conv}} w-\overline{\lim } A\left(t, x(t)+\lambda_{n} y(t)\right) \\
& \cong A(t, x(t)+\lambda y(t)) \text { a. e. }
\end{aligned}
$$

the last inclusion following from hypothesis $H(A)(2)$ and the fact that $A(\cdot, \cdot)$ is $P_{w k c}\left(X^{*}\right)$-valued being maximal monotone by lemma 3.1. So $f \in \hat{\alpha}(\lambda)$. Clearly $f \in C$. Hence $f \in \hat{\alpha}(\lambda) \cap C \Rightarrow \lambda \in \hat{\alpha}^{-}(C) \Rightarrow \hat{\alpha}^{-}(C)$ is closed $\Rightarrow \hat{\alpha}(\cdot)$ is u. s. c. from $[0,1]$ into $L^{2}\left(X^{*}\right)_{w}$. Then from lemma 3.1 we get that $\hat{A}(\cdot)$ is maximal monotone and furthermore, from hypothesis $H(A)(4)$, we have that it is coercive. Therefore $E+A$ is a maximal monotone coercive map, hence surjective. Thus we conclude that $(*)^{\prime}$ has a solution $x(\cdot) \in W(T)$ and uniqueness follows easily from the monotonicity of $A(t, \cdot)$.

Q.E.D.

Now we are ready for our first theorem. It concerns the existence of solutions for the following multivalued Cauchy problem:

$$
\left\{\begin{array}{l}
\dot{x}(t) \in-A(t, x(t))+F(t, x(t)) \text { a.e. } \\
x(0)=\xi
\end{array}\right\}(*)
$$

For this we will need the following hypothesis on the orientor field $F(t, x)$. $H(F): \quad F: T \times H \rightarrow P_{f}(H)$ is a multifunction s.t. 
(1) $(t, x) \rightarrow F(t, x)$ is graph measurable,

(2) $x \rightarrow F(t, x)$ is 1.s.c.,

(3) $|F(t, x)|=\sup \{|v|: v \in F(t, x)\} \leqq a_{1}(t)+b_{1}|x|$ a.e. with $a_{1}(\cdot) \in L_{+}^{2}, b_{1}>0$.

We will denote the solution set of $\left(^{*}\right)$ by $S(\xi) \cong W(T)$

TheOREM 3.4. If hypotheses $H(A), H(F)$ hold and $\xi \in H$, then $S(\xi) \subseteq W(T)$ is nonempty.

Proof. We will start by deriving some a priori bounds for the elements in $S(\xi)$. So let $x(\cdot) \in S(\xi)$. Then by definition there exists $f \in S_{F(\cdot, x(\cdot))}^{2}$ s.t.

$$
\left\{\begin{array}{l}
\dot{x}(t) \in-A(t, x(t))+f(t) \text { a.e. } \\
x(0)=\xi
\end{array}\right\}
$$

We have:

$$
\langle\dot{x}(t), x(t)\rangle \leqq \sigma(x(t) ;-A(t, x(t)))+(f(t), x(t)) \quad \text { a. e. }
$$

where $\sigma(x(t) ;-A(t, x(t)))=\sup \left\{\left\langle-v^{*}, x(t)\right\rangle: v^{*} \in A(t, x(t))\right\}$. Hence

$$
\begin{aligned}
& \langle\dot{x}(t), x(t)\rangle+r(x(t) ; A(t, x(t))) \leqq(f(t), x(t)) \quad \text { a.e. } \\
\Longrightarrow & \langle\dot{x}(t), x(t)\rangle+c\|x(t)\|^{2} \leqq \frac{\epsilon}{2}\|f(t)\|_{*}^{2}+\frac{1}{2 \epsilon}\|x(t)\|^{2} \quad \text { a. e., } \epsilon>0 .
\end{aligned}
$$

Let $\epsilon=1 / 2 c>0$ and integrate the above inequality. We get

$$
\begin{aligned}
|x(t)|^{2} \leqq \frac{\beta^{2}}{4 c} \int_{0}^{t}|f(s)|^{2} d s & \leqq \frac{\beta^{2}}{4 c} \int_{0}^{t}\left(2 a_{1}(s)^{2}+2 b_{1}^{2}|x(s)|^{2}\right) d s \\
& =\frac{\beta^{2}}{2 c} \int_{0}^{t}\left(a_{1}(s)^{2}+b_{1}^{2}|x(s)|^{2}\right) d s
\end{aligned}
$$

where $\beta>0$ is such that $\|\cdot\|_{*} \leqq \beta|\cdot|$ (it exists since $H \subset X^{*}$ continuously). Invoking Gronwall's inequality we get $M_{1}>0$ s.t. for all $t \in T$ and all $x(\cdot) \in S(\xi)$, we have

$$
|x(t)| \leqq M_{1}
$$

Next from (1), with $\epsilon=1 / c$, we get

$$
\frac{1}{2} \frac{d}{d t}|x(t)|^{2}+\frac{c}{2}\|x(t)\|^{2} \leqq \frac{1}{2 c}\|f(t)\|_{*}^{2} \quad \text { a.e. }
$$

Integrating, we have

$$
\begin{aligned}
|x(b)|^{2}+c \int_{0}^{b}\|x(s)\|^{2} d s & \leqq \frac{\beta^{2}}{c} \int_{0}^{b}|f(s)|^{2} d s+|\xi|^{2} \\
& \leqq \frac{\beta^{2}}{c} \int_{0}^{b}\left(2 a_{1}(s)^{2}+2 b_{1}^{2} M_{1}^{2}\right) d s+|\xi|^{2}
\end{aligned}
$$




$$
\begin{aligned}
& \Longrightarrow\|x\|_{L^{2}(X)}^{2} \leqq \frac{2 \beta^{2}}{c^{2}}\left(\left\|a_{1}\right\|_{2}^{2}+b_{1}^{2} M_{1}^{2} b\right)+\frac{|\xi|^{2}}{c}=M_{2}^{2} \\
& \Longrightarrow\|x\|_{L^{2}(X)} \leqq M_{2}, M_{2}>0 \text { independent of } x(\cdot) \in S(\xi)
\end{aligned}
$$

Finally let $h \in L^{2}(X)$. We have

$$
\begin{aligned}
\langle\dot{x}(t), h(t)\rangle & \leqq \sigma(h(t) ;-A(t, x(t)))+(f(t), h(t)) \quad \text { a.e. } \\
& \leqq\|h(t)\| \cdot|A(t, x(t))|+\|f(t)\|_{*}\|h(t)\| \quad \text { a.e. } \\
& \leqq\|h(t)\| \cdot|A(t, x(t))|+\beta|f(t)| \cdot\|h(t)\| \quad \text { a.e. } \\
& \leqq\left(a(t)+b^{\prime}\|x(t)\|+\beta a_{1}(t)+\beta b_{1} M_{1}\right)\|h(t)\| \quad \text { a.e. }
\end{aligned}
$$

by using hypotheses $H(A)(5), H(F)$ (3) and bound (2) above. So

$$
((\dot{x}, h))_{0}=\int_{0}^{b}\langle\dot{x}(t), h(t)\rangle d t \leqq\left(\|a\|_{2}+\beta\left\|a_{1}\right\|_{2}+\beta b_{1} M_{1} b+b M_{2}\right)\|h(t)\|_{L^{2}(X)}
$$

by using the Cauchy-Schwartz inequality and bound (3) above (recall that by $((\cdot, \cdot))_{0}$ we denote the duality brackets for the pair $\left.\left(L^{2}(X), L^{2}\left(X^{*}\right)\right)\right)$. Since $h \in L^{2}(X)$ was arbitrary we deduce that for all $x(\cdot) \in S(\xi)$ we have

$$
\|\dot{x}\|_{L^{2}\left(X^{*}\right)} \leqq\left(\|a\|_{2}+\beta\left\|a_{1}\right\|_{2}+\beta b_{1} M_{1} b+b M_{2}\right)=M_{3}
$$

From (3) and (4) above, we see that for all $x(\cdot) \in S(\xi)$, we have

$$
\|x\|_{W(T)} \leqq\left(M_{2}^{2}+M_{3}^{2}\right)^{1 / 2}=M_{4}
$$

Next let $\hat{F}: T \times H \rightarrow P_{f}(H)$ be defined by

$$
\hat{F}(t, x)=\left\{\begin{array}{lll}
F(t, x) & \text { if } & |x| \leqq M_{1} \\
F\left(t, \frac{M_{1} x}{|x|}\right) & \text { if } & |x|>M_{1}
\end{array}\right.
$$

Note that $\hat{F}(t, x)=F\left(t, p_{M_{1}}(x)\right)$, where $p_{M_{1}}: H \rightarrow H$ is the $M_{1}$-radial retraction. Recalling that $p_{M_{1}}(\cdot)$ is Lipschitz continuous, we easily see that $(t, x) \rightarrow \hat{F}(t, x)$ is graph measurable, while $x \rightarrow \hat{F}(t, x)$ is 1 .s.c. and in addition $|\hat{F}(t, x)|=$ $\sup \{|v|: v \in \hat{F}(t, x)\} \leqq a_{1}(t)+b_{1} M_{1}=\hat{a}_{1}(t)$ a. e. with $\hat{a}_{1}(\cdot) \in L_{+}^{2}$.

Let $B\left(M_{4}\right)=\left\{x \in W(T):\|x\|_{W(T)} \leqq M_{4}\right\}$, where $M_{4}$ is as in (5) above. By $B\left(M_{4}\right)_{w}$ we will denote the set $B\left(M_{4}\right)$ endowed with the relative weak topology in $W(T)$. From theorem 3, p. 434 of Dunford-Schwartz [10], we know that $B\left(M_{4}\right)_{w}$ is compact, metrizable. Let $R: B\left(M_{4}\right)_{w} \rightarrow P_{f}\left(L^{1}(H)\right)$ be defined by $R(x)$ $=S_{\hat{F}}^{1}(\cdot, x(\cdot))$. From theorem 4.1 of $[17]$ and the fact that $\hat{F}(t, \cdot)$ is 1 .s.c., we get that $R(\cdot)$ is l.s.c.. Apply Fryszkowski's continuous selection theorem (see [13]), to get $r: B\left(M_{4}\right)_{w} \rightarrow L^{1}(H)$ continuous s.t. $r(x) \in R(x)$ for all $x \in B\left(M_{4}\right)$. Then consider the following evolution inclusion: 


$$
\left\{\begin{array}{l}
\dot{y}(t) \in-A(t, y(t))+r(x)(t) \text { a.e. } \\
y(0)=\xi
\end{array}\right\}
$$

Let $\eta(x)(\cdot) \in W(T)$ be the unique solution of the above inclusion. The existence and uniqueness of $\eta(x)(\cdot)$, follow from lemma 3.3. Then consider the map $x \rightarrow \eta(x)$ from $B\left(M_{4}\right)_{w}$ into itself. Our claim is that $\eta(\cdot)$ is continuous. Since $B\left(M_{4}\right)_{w}$ is compact, metrizable, it is enough to show that Gr $\eta$ is sequentially closed in $B\left(M_{4}\right)_{w} \times B\left(M_{4}\right)_{w}$. So let $\left[x_{n}, y_{n}\right] \rightarrow[x, y]$ in $B\left(M_{4}\right)_{w} \times B\left(M_{4}\right)_{w}$, with $y_{n}=\eta\left(x_{n}\right) \quad n \geqq 1$. Let $\Gamma_{n}(t)=\left\{x * \in A\left(t, y_{n}(t)\right): \dot{y}_{n}(t)+x^{*}=r\left(x_{n}\right)(t)\right\}$. Then for every $n \geqq 1$, we have $\Gamma_{n}(t) \neq \emptyset$ for all $t \in T \backslash N_{n}, \lambda\left(N_{n}\right)=0$, with $\lambda(\cdot)$ being the Lebesgue measure on $T$. Furthermore

$$
\Gamma_{n}(t)=\bigcap_{k \geqq 1}\left\{x^{*} \in X^{*}:\left\langle x^{*}, z_{k}\right\rangle \leqq \sigma\left(z_{k} ; A\left(t, y_{n}(t)\right), y_{n}(t)+x^{*}=r\left(x_{n}\right)(t)\right\}\right.
$$

where $\left\{z_{k}\right\}_{k \geqq 1}$ is dense in $X$. From lemma 3.2 we know that $t \rightarrow A\left(t, y_{n}(t)\right)$ is Lebesgue measurable. So $t \rightarrow \sigma\left(z_{k} ; A\left(t, y_{n}(t)\right)\right)$ is Lebesgue measurable. Thus $\operatorname{Gr} \Gamma_{n} \in \mathcal{L} \times B\left(X^{*}\right)$, with $\mathcal{L}$ being the Lebesgue $\sigma$-field of $T$. Apply Aumann's selection theorem (see for example Wagner [21], theorem 5.10), to get $x_{n}^{*}: T \rightarrow X^{*}$ $n \geqq 1$ measurable s. t. $x_{n}^{*}(t) \in \Gamma_{n}(t) \subseteq A\left(t, y_{n}(t)\right)$ a. e.. Clearly from hypothesis $H(A)$ (5) $x_{n}^{*}(\cdot) \in L^{2}\left(X^{*}\right) n \geqq 1$. Then we have

$$
\left(\left(\dot{y}_{n}, y_{n}-y\right)\right)_{0}+\left(\left(x_{n}^{*}, y_{n}-y\right)\right)_{0}=\left(\left(r\left(x_{n}\right), y_{n}-y\right)\right)_{0}
$$

Note that $y_{n} \stackrel{s}{\rightarrow} y$ in $L^{2}(H)$ (since $W(T) \hookrightarrow L^{2}(H)$ compactly, see Zeidler [22], p. 450) and $r\left(x_{n}\right) \stackrel{s}{\rightarrow} r(x)$ in $L^{1}(H)$. But $\left|r\left(x_{n}\right)\right|,|r(x)| \leqq \hat{a}_{1}(t)$ with $\hat{a}_{1}(\cdot) \in L_{+}^{2}$. So $r\left(x_{n}\right) \stackrel{s}{\rightarrow} r(x)$ in $L^{2}(H)$. Hence

$$
\begin{aligned}
\left(\left(r\left(x_{n}\right), y_{n}-y\right)\right)_{0} & =\int_{0}^{b}\left\langle r\left(x_{n}\right)(t), y_{n}(t)-y(t)\right\rangle d t \\
& =\int_{0}^{b}\left(r\left(x_{n}\right)(t), y_{n}(t)-y(t)\right) d t \longrightarrow 0 \quad \text { as } n \rightarrow \infty .
\end{aligned}
$$

Also from the integration by parts formula for functions in $W(T)$ (see Zeidler [22], proposition 23.23 (iv), p. 423), we get

and

$$
\left(\left(\dot{y}_{n}, y_{n}-y\right)\right)_{0}=\frac{1}{2}\left|y_{n}(b)-y(b)\right|^{2}+\left(\left(\dot{y}, y_{n}-y\right)\right)_{0}
$$

$$
\frac{1}{2}\left|y_{n}(b)-y(b)\right|^{2}+\left(\left(\dot{y}, y_{n}-y\right)\right)_{0} \longrightarrow 0 \quad \text { as } n \rightarrow \infty \text {. }
$$

Hence we have

$$
\left(\left(x_{n}^{*}, y_{n}-y\right)\right)_{0} \longrightarrow 0 \quad \text { as } n \rightarrow \infty .
$$

Now because of $H(A)$ (5), by passing to a subsequence if necessary, we may assume that $x_{n}^{*} \underset{w}{\rightarrow} x^{*}$ in $L^{2}\left(X^{*}\right)$. Recall that $\hat{A}(z)=S_{A(\cdot, z(\cdot))}^{2}$ is maximal monotone 
from $L^{2}(X)$ into $P_{f c}\left(L^{2}\left(X^{*}\right)\right.$ ) (see the proof of lemma 3.3). So from proposition 8 of Browder-Hess [7] (see also Kenmochi [14], proposition 1.6) is pseudomonotone. Then proposition 7 of Browder-Hess [7] (see also Kenmochi [14], proposition 1.1) tells us that $x^{*} \in \hat{A}(y)$. Thus if $h \in L^{2}(X)$, we have

$$
\begin{aligned}
& \left(\left(\dot{y}_{n}, h\right)\right)_{0}+\left(\left(x_{n}^{*}, h\right)\right)_{0}=\left(\left(r\left(x_{n}\right), h\right)\right)_{0} \\
\Longrightarrow & ((\dot{y}, h))_{0}+\left(\left(x^{*}, h\right)\right)_{0}=((r(x), h))_{0} \text { as } n \rightarrow \infty, \\
\Longrightarrow & \dot{y}(t)+x^{*}(t)=r(x)(t) \text { a. e. } \\
\Longrightarrow & \dot{y}(t) \in-A(t, y(t))+r(x)(t) \text { a. e. and } y(0)=\xi \\
\Longrightarrow & y=\eta(x) \\
\Longrightarrow & \eta(\cdot) \text { is continuous as claimed. }
\end{aligned}
$$

Then we can apply the Schauder-Tichonov fixed point theorem, and get $\hat{x} \in$ $B\left(M_{4}\right)$ s. t. $\hat{x}=\eta(\hat{x})$. Clearly then $\hat{x} \in W(T)$ solves $\left(^{*}\right)$ for the orientor field $\hat{F}(t, x)$. As in the beginning of the proof, using the definition of $\hat{F}(t, x)$, we can easily establish that $|\hat{x}(t)| \leqq M_{1}$ for all $t \in T \Rightarrow \hat{F}(t, \hat{x}(t))=F(t, \hat{x}(t)) \Rightarrow \hat{x}(\cdot) \in W(T)$ is the desired solution of $\left(^{*}\right)$; i.e. $S(\xi) \subseteq W(T)$ is nonempty. Q.E, D.

Now we will produce a continuous selector for the multifunction $\xi \rightarrow S(\xi)$ going from $H$ into $2^{C(T, H)} \backslash\{\emptyset\}$. Our proof follows that of Colombo et al [9] and Staicu [20]. For this we will need the following stronger hypothesis on the orientor field $F(t, x)$.

$H\left(F_{1}\right): \quad F: T \times H \rightarrow P_{f c}(H)$ is a multifunction s.t.

(1) $t \rightarrow F(t, x)$ is measurable,

(2) $h\left(F(t, x), F\left(t, x^{\prime}\right)\right) \leqq k(t)\left|x-x^{\prime}\right|$ a.e. with $k(\cdot) \in L_{+}^{1}, x, x^{\prime} \in H$,

(3) $|F(t, x)| \leqq a_{1}(t)+b_{1}|x|$ a.e. with $a_{1}(\cdot) \in L_{+}^{2}, b_{1}>0$.

Remark. Note that Staicu [20] assumed that $(t, x) \rightarrow F(t, x)$ is jointly measurable. However from theorem 3.3 of [18], we know that this is immediately implied by $H(F)_{1}(1)$ and (2) above. Also if $k(\cdot) \in L_{+}^{2}$, then instead of $H(F)_{1}$ (3), we can have $|F(t, 0)| \leqq a_{1}(t)$ a. e., $a_{1}(\cdot) \in L_{+}^{2}$.

THEOREM 3.5. If hypotheses $H(A)$ and $H(F)_{1}$ hold, then there exists $\phi: H \rightarrow C(T, H)$ continuous map s.t. $\phi(\xi) \in S(\xi)$ for all $\xi \in H$.

Proof. Let $x_{0}(\xi) \in W(T) \hookrightarrow C(T, H)$ be the unique solution of the evolution inclusion

$$
\left\{\begin{array}{l}
\dot{x}(t) \in-A(t, x(t)) \text { a.e. } \\
x(0)=\xi
\end{array}\right\}
$$

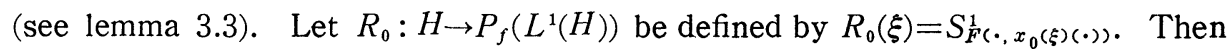
$R_{0}(\cdot)$ is 1 .s.c. (in fact Hausdorff continuous; see theorem 4.5 of [17]). Apply 
theorem 3 of Bressan-Colombo [5], to get $r_{0}: H \rightarrow L^{1}(H)$ continuous map s.t. $r_{0}(\xi) \in R_{0}(\xi)$ for all $\xi \in H$. Then consider the following multivalued Cauchy problem :

$$
\left\{\begin{array}{l}
\dot{x}(t) \in-A(t, x(t))+r_{0}(\xi)(t) \text { a.e. } \\
x(0)=\xi
\end{array}\right\}
$$

Again lemma 3.3 tells us that this has a unique solution $x_{1}(\xi)(\cdot) \in W(T) \hookrightarrow$ $C(T, H)$.

By induction we will construct sequences $\left\{r_{n}(\xi)(\cdot)\right\}_{n \geqq 1} \leqq L^{2}(H) \hookrightarrow L^{1}(H)$ and $\left\{x_{n}(\xi)(\cdot)\right\}_{n \geqq 1} \leqq W(T) \hookrightarrow C(T, H)$ s, t.

(i) $\xi \rightarrow r_{n}(\xi)$ is continuous from $H$ into $L^{1}(H)$.

(ii) $r_{n}(\xi)(t) \in F\left(t, x_{n}(\xi)(t)\right)$ a.e. for every $\xi \in H$,

(iii) $\left|r_{n}(\xi)(t)-r_{n-1}(\xi)(t)\right| \leqq k(t) \beta_{n}(\xi)(t)$ a.e.

(iv) $x_{n}(\xi)(\cdot) \in W(T) \hookrightarrow C(T, H)$ solves the multivalued Cauchy problem

$$
\left\{\begin{array}{l}
\dot{x}(t) \in-A(t, x(t))+r_{n-1}(\xi)(t) \text { a.e. } \\
x(0)=\xi
\end{array}\right\}
$$

where $\beta_{n}(\xi)(t)=2 \int_{0}^{t} \lambda(\xi)(s) \frac{(\theta(t)-\theta(s))^{n-1}}{(n-1) !} d s+2 b\left(\sum_{\imath=0}^{n} \frac{\epsilon}{2^{2+1}}\right) \times \frac{\theta(t)^{n-1}}{(n-1) !}, \epsilon>0$ and $\lambda(\xi)(t)$ $=a_{1}(t)+b_{1}\left|x_{0}(\xi)(t)\right|$, while $\theta(t)=\int_{0}^{t} k(s) d s$. Clearly $\xi \rightarrow \beta_{n}(\xi)$ and $\xi \rightarrow \lambda(\xi)$ are both continuous from $H$ into $L^{1}$.

Suppose we have defined $\left\{r_{i}\right\}_{l=0}^{n}$ and $\left\{x_{i}\right\}_{l=0}^{n}$. Let $x_{n+1}(\xi)(\cdot)$ be the unique solution of $\dot{x}(t) \in-A(t, x(t))+r_{n}(\xi)(t)$ a. e., $x(0)=\xi$ (see lemma 3.3). We have:

$$
\begin{aligned}
& \left\langle\dot{x}_{n+1}(\xi)(t)-\dot{x}_{n}(\xi)(t), x_{n+1}(\xi)(t)-x_{n}(\xi)(t)\right\rangle \\
\leqq & \left\langle r_{n}(\xi)(t)-r_{n-1}(\xi)(t), x_{n+1}(\xi)(t)-x_{n}(\epsilon)(t)\right\rangle \quad \text { a. e., } \\
\Longrightarrow & \frac{1}{2} \frac{d}{d t}\left|x_{n+1}(\xi)(t)-x_{n}(\xi)(t)\right|^{2} \leqq\left|r_{n}(\xi)(t)-r_{n-1}(\xi)(t)\right| \cdot\left|x_{n+1}(\xi)(t)-x_{n}(\xi)(t)\right| \quad \text { a. e. } \\
\Longrightarrow & \left|x_{n+1}(\xi)(t)-x_{n}(\xi)(t)\right|^{2} \leqq 2 \int_{0}^{t}\left|r_{n}(\xi)(s)-r_{n-1}(\xi)(s)\right| \cdot\left|x_{n+1}(\xi)(s)-x_{n}(\xi)(s)\right| d s .
\end{aligned}
$$

Invoking lemma A.5 p. 157 of Brezis [6], we get

$$
\begin{aligned}
& \left|x_{n+1}(\xi)(t)-x_{n}(\xi)(t)\right| \leqq 2 \int_{0}^{t}\left|r_{n}(\xi)(s)-r_{n-1}(\xi)(s)\right| d s, \quad t \in T, \\
\leqq & 2 \int_{0}^{t} k(s) \beta_{n}(\xi)(s) d s \\
\leqq & 2 \int_{0}^{t} k(s) \int_{0}^{s} \lambda(\xi)(v) \frac{(\theta(s)-\theta(v))^{n-1}}{(n-1) !} d v d s+2 b\left(\sum_{\imath=0}^{n} \frac{\epsilon}{2^{2+1}}\right) \int_{0}^{t} k(s) \frac{\theta(s)^{n-1}}{(n-1) !} d s
\end{aligned}
$$




$$
\begin{aligned}
& =2 \int_{0}^{t} \lambda(\xi)(s) \int_{s}^{t} k(v) \frac{(\theta(v)-\theta(s))^{n-1}}{(n-1) !} d v d s+2 b\left(\sum_{\imath=0}^{n} \frac{\epsilon}{2^{2+1}}\right) \frac{\theta(t)^{n}}{n !} \\
& =2 \int_{0}^{t} \lambda(\xi)(s) \int_{s}^{t} \frac{d}{d v} \frac{(\theta(v)-\theta(s))^{n}}{n !} d v d s+2 b\left(\sum_{\imath=0}^{n} \frac{\epsilon}{2^{2+1}}\right) \frac{\theta(t)^{n}}{n !} \\
& =2 \int_{0}^{t} \lambda(\xi)(s) \frac{(\theta(t)-\theta(s))^{n}}{n !} d s+2 b\left(\sum_{\imath=0}^{n} \frac{\epsilon}{2^{\imath+1}}\right) \frac{\theta(t)^{n}}{n !}<\beta_{n+1}(\xi)(t) \quad \text { a. e. }
\end{aligned}
$$

From hypothesis $H(F)_{1}(2)$, we have

$$
\begin{aligned}
d\left(r_{n}(\xi)(t), F\left(t, x_{n+1}(\xi)(t)\right.\right. & \leqq k(t)\left|x_{n}(\xi)(t)-x_{n+1}(\xi)(t)\right| \\
& <k(t) \beta_{n+1}(\xi)(t) \quad \text { a.e. }
\end{aligned}
$$

Let $R_{n+1}: H \rightarrow 2^{L^{1}(H)}$ be the multifunction defined by

$$
R_{n+1}(\xi)=\left\{z \in S_{F\left(\cdot, x_{n+1}(\xi)(\cdot)\right)}^{1}:\left|z(t)-r_{n}(\xi)(t)\right|<k(t) \beta_{n+1}(\xi)(t) \text { a.e. }\right\} .
$$

First we will show that $R_{n+1}(\cdot)$ has nonempty values. From (2) above we have that

$$
d\left(r_{n}(\xi)(t), F\left(t, x_{n+1}(\xi)(t)\right)\right)<k(t) \beta_{n+1}(\xi)(t) \quad \text { a.e. }
$$

Let $H_{n+1}(\xi)(t)=\left\{v \in F\left(t, x_{n+1}(\xi)(t)\right):\left|r_{n}(\xi)(t)-v\right|<k(t) \beta_{n+1}(\xi)(t)\right\}$. Clearly $H_{n+1}(\xi)(\cdot)$ is nonempty valued and graph measurable, so Aumann's selection theorem (see Wagner [21], theorem 5.10) guarantees that there exists $z: T \rightarrow H$ a measurable selector of $H_{n+1}(\xi)(\cdot)$. Clearly then $z(\cdot) \in R_{n+1}(\xi)$. So $R_{n+1}(\cdot)$ has nonempty values. Next we will show that $R_{n+1}: H \rightarrow 2^{L^{1}(H)} \backslash\{\emptyset\}$ is 1 .s.c.. To this end let $R_{n+1}^{1}(\xi)=S_{F(}^{1}\left(, x_{n+1}(\xi)(\cdot)\right)$ and $R_{n+1}^{2}(\xi)=\left\{v \in L^{2}(H):\left|v(t)-r_{n}(\xi)(t)\right|<k(t)\right.$ $\beta_{n+1}(\xi)(t)$ a.e.\}. From theorem 4.1 of [17] we know that $R_{n+1}^{1}(\cdot)$ is 1 .s.c.. We claim that $G r R_{n+1}^{2}$ is open in $H \times L^{1}(H)$. We will show that $\left[G r R_{n+1}^{2}\right]^{c}$ is closed in $H \times L^{1}(H)$. But $\left[G r R_{n+1}^{2}\right]^{c}=\left\{[\xi, w] \in H \times L^{1}(H):\left|w(t)-r_{n}(\xi)(t)\right| \geqq k(t) \beta_{n+1}(\xi)(t)\right.$ a.e.\} and this set is clearly closed since both $\xi \rightarrow r_{n}(\xi)$ and $\xi \rightarrow \beta_{n+1}(\xi)$ are continuous from $H$ into $L^{1}$. Hence indeed $R_{n+1}^{2}(\cdot)$ has" an open graph. Then from lemma 4.2 of Flytzanis-Papageorgiou [12], we have that $\xi \rightarrow R_{n+1}^{1}(\xi) \cap R_{n+1}^{2}(\xi)=$ $R_{n+1}(\xi)$ is 1 .s.c.. Also it is clear that $R_{n+1}(\cdot)$ has decomposable values; i.e. if $z_{1}, z_{2} \in R_{n+1}(\xi)$ and $C \in \mathcal{L}$, then clearly $\chi_{c} z_{1}+\chi_{c} z_{2} \in R_{n+1}(\xi)$. So we can apply theorem 3 of Bressan-Colombo [5] to get $r_{n+1}: H \rightarrow L^{1}(H)$ a continuous map s.t. $r_{n+1}(\xi) \in R_{n+1}(\xi)$ for all $\xi \in H$. This completes the inductive construction of the sequences $\left\{r_{n}(\xi)(\cdot)\right\}_{n \geqq 1} \subseteq L^{2}(H) \subset L^{1}(H)$ and $\left\{x_{n}(\xi)(\cdot)\right\}_{n \geqq 1} \subseteq W(T) \hookrightarrow C(T, H)$, satisfying $(1) \rightarrow$ (iv) above.

Now that we have those two sequences, we proceed as follows:

$$
\int_{0}^{b}\left|r_{n}(\xi)(t)-r_{n-1}(\xi)(t)\right| d t<\beta_{n+1}(\xi)(b) \quad(\text { see }(1) \text { above })
$$




$$
\begin{aligned}
& \leqq \int_{0}^{b} \lambda(\xi)(t) \frac{(\theta(b)-\theta(t))^{n}}{n !} d t+b \varepsilon \frac{\theta(b)^{n}}{n !} \\
& \leqq \frac{\theta(b)^{n}}{n !}\left[\|\lambda(\xi)(\cdot)\|_{1}+b \epsilon\right]
\end{aligned}
$$

Note that since $\xi \rightarrow \lambda(\xi)$ is continuous from $H$ into $L^{1}$, it is locally bounded. So from (3) above we deduce that $\left\{r_{n}(\xi)(\cdot)\right\}_{n \geqq 1}$ is an $L^{1}(H)$-Cauchy sequence, locally uniformly in $\xi \in H$.

Also once again from (1) above we get

$$
\left\|x_{n+1}(\xi)-x_{n}(\xi)\right\|_{C(T, H)} \leqq 2\left\|r_{n}(\xi)-r_{n-1}(\xi)\right\|_{L^{1}(H)} \leqq \frac{\theta(b)^{n}}{n !}\left[\|\lambda(\xi)(\cdot)\|_{1}+b \epsilon\right]
$$

$\Longrightarrow\left\{x_{n}(\xi)(\cdot)\right\}_{n \geqq 1}$ is Cauchy in $C(T, H)$, locally uniformly in $\xi \in H$.

Then we have $r_{n}(\xi) \stackrel{s}{\rightarrow} r(\xi)$ in $L^{1}(H)$ and $x_{n}(\xi) \stackrel{s}{\rightarrow} x(\xi)$ in $C(T, H)$. Both limits are continuous in $\xi$. For each $\xi \in H$, let $\hat{x}(\xi) \in W(T) \hookrightarrow C(T, H)$ be the unique solution of

$$
\left\{\begin{array}{l}
\dot{y}(t) \in-A(t, y(t))+r(\xi)(t) \text { a.e. } \\
y(0)=\xi
\end{array}\right\}
$$

We have been using the monotonicity of $A(t, \cdot)$ and lemma A.5, p. 157 of Brezis [6].

$$
\begin{aligned}
& \left|x_{n}(\xi)(t)-\hat{x}(\xi)(t)\right|^{2} \leqq 2 \int_{0}^{t}\left|r_{n}(\xi)(s)-r(\xi)(s)\right| \cdot\left|x_{n}(\xi)(s)-\hat{x}(\xi)(s)\right| d s \\
& \Longrightarrow\left\|x_{n}(\xi)-\hat{x}(\xi)\right\|_{C(T, H)} \leqq\left\|r_{n}(\xi)-r(\xi)\right\|_{L^{1}(H)} \longrightarrow 0 \quad \text { as } n \rightarrow \infty \\
& \Longrightarrow x(\xi)=\hat{x}(\xi)
\end{aligned}
$$

Then $\phi: \xi \rightarrow x(\xi)$ is the desired continuous selector of $S(\xi)$ Q.E.D.

\section{Periodic solutions.}

In this section we use theorem 3.5 together with the viability result of [3] (theorem 1), to establish the existence of periodic solutions for a class of time invariant, nonlinear evolution inclusions. Our result extends proposition 4.1 of Staicu [20], who assumed that $A \equiv 0$.

Here we will assume that $\left(X, H, X^{*}\right)$ is an evolution triple of Hilbert spaces with all embedding being compact. We consider the following boundary value problem :

$$
\left\{\begin{array}{l}
\dot{x}(t)+A x(t) \in F(x(t)) \text { a.e. } \\
x(0)=x(b)=\xi \in K
\end{array}\right\}
$$

The hypotheses on the data of $(* *)$ are the following: 
$H(A)_{1}: A: X \rightarrow X^{*}$ is an operator s.t.

(1) $A(\cdot)$ is monotone, hemicontinuous,

(2) $\langle A(x), x\rangle \geqq c\|x\|^{2}$ with $c>0$,

(3) $\|A(x)\|_{*} \leqq a(1+\|x\|)$ with $a>0$.

$H(K): K \subseteq H$ is a nonempty, bounded, closed and convex set.

$H(F)_{2}: \quad F: K \rightarrow P_{f c}\left(X^{*}\right)$ is a multifunction s.t.

(1) $x \rightarrow F(x)$ is u.c.s.,

(2) $|F(x)|=\sup \{|v|: v \in F(x)\} \leqq M_{0}$

$H_{\tau}$ : For every $x \in K \cap X$, we have $(-A(x)+F(x)) \subseteq T_{K}^{\prime}(x) \neq \emptyset$, where $T_{K}^{\prime}(x)$ denotes the Bouligand tangent cone to $K$ at $x$ in the space $X^{*}$; i.e. $T_{K}^{\prime}(x)=$ $\left\{h^{*} \in X^{*}: \lim _{\lambda \downarrow 0} \frac{d_{K}^{*}\left(x+\lambda h^{*}\right)}{\lambda}=0\right\}$ where $d_{K}^{*}(\cdot)$ is the distance function from $K$ in the space $X^{*}$.

Then we have the following result concerning $\left({ }^{* *}\right)$ :

THEOREM 4.1. If hypotheses $H(A)_{1}, H(F)_{2}, H(K)$ and $H_{\tau}$ hold, then problem (**) admits a solution.

Proof. From theorem 1 of [3], we know that the evolution inclusion under consideration admits a viable trajectory (i. e. there exists $x(\cdot) \in W(T) \subset C(T, H)$ solution of $(* *)$ s.t. $x(t) \in K$ for all $t \in T)$. Let $\phi: H \rightarrow C(T, H)$ be a continuous map s.t. $\phi(\xi) \in S(\xi)=$ solution set of $\dot{x}(t)+A(x(t)) \in F(x(t))$ a.e., $x(0)=\xi\}$. Its existence is guaranteed by theorem 3.5. Define $e: K \rightarrow K$ by $\epsilon(\xi)=\phi(\xi)(b)$. Clearly $e(\cdot)$ is continuous. Also since by hypothesis $H(K), K$ is bounded in $H$, from the a priori estimation that we did in the beginning of the proof of theorem 3.4, we have that $N=\bar{\bigcup}_{\xi \in K} S(\xi)^{W(T)}$ is bounded in $W(T)$. But from Nagy [16], we know that $W(T) \hookrightarrow C(T, H)$ compactly. So we deduce that $N \leqq C(T, H)$ is compact $\Rightarrow N(b)=\{y(b): y \in N\} \subseteq K$ is compact. Note that $e(K) \subseteq N(b)$. Then from Schauder's fixed point theorem, we get $\hat{\xi} \in K$ s.t. $e(\hat{\xi})=\hat{\xi} \Rightarrow \phi(\hat{\xi})(b)=\hat{\xi}=\phi(\hat{\xi})(0) \Rightarrow$ $\phi(\hat{\xi})(\cdot)$ is the desired periodic solution.

Q.E.D.

Remark - Our result also extends theorem 4, p. 237 of Aubin-Cellina [2], which deals only with finite dimensional differential inclusions.

We can use this result to establish the existence of periodic trajectories for closed loop (feedback) control systems. Let $Z$ be a separable reflexive Banach space, modelling the control space and consider the following system:

$$
\left\{\begin{array}{ll}
\dot{x}(t)+A x(t)= & B(x(t)) u(t) \text { a.e. } \\
x(0)=x(b), & u(t) \in U(x(t)) \text { a.e. } \\
& u(\cdot)=\text { measurable }
\end{array}\right\} \quad(* *)^{\prime}
$$

We will need the following hypotheses on the data of $(* *)^{\prime}$. 
$H(B): \quad B: K \rightarrow \mathcal{L}(Z, H)=$ bounded linear operators from $Z$ into $H\}, B(K)$ is bounded and $h \rightarrow B(h)^{*}$ is continuous from $K$ into $\mathcal{L}(H, Z)$ with the stuong operator topology.

$H(U): \quad U: K \rightarrow P_{f c}(Z)$ is an u.c.s. multifunction $|U(x)| \leqq \eta, \eta>0$.

$H_{\tau}^{\prime}:$ For every $x \in K \cap X, C(x)=\left\{u \in U(x):(-A(x)+B(x) u) \cap T_{K}^{\prime}(x) \neq \emptyset\right\} \neq \emptyset$.

Then using theorem 4.1 we can have the following existence theorem concerning $(* *)$. Its proof is the same as that of theorem 2 in [3] and so is omitted.

ThEOREM 4.2. If hypotheses $H(A)_{1}, H(B), H(U), H(K)$ and $H_{T}^{\prime}$ hold, then (**) admits a solution.

Acknowledgement: The author wishes to thank the referee for his corrections and remarks.

\section{REFERENCES}

[1] N.U. Ahmed, "Existence of optimal controls for a class of systems governed by differential inclusions on a Banach space" J. Optim. Th. Appl 50 (1986), pp. 213-237.

[2] J.-P. Aubin ANd A. Cellina, "Differential Inclusions" Springer, Berlin (1984).

[3] E. Avgerinos and N.S. Papageorgiou, "Viable solutions for evolution inclusions" Yokohama Math. Jour. 37 (1990), pp. 101-108.

[4] E. Avgerinos And N.S. Papageorgiou, "Optimal control and relaxation for a class of nonlinear distributed parameter systems" Osaka J. Math 27 (1990), pp. 745-767.

[5] A. Bressan and G. Colombos, "Extensions and selections of maps with decomposable values" Studia Math 90 (1988), pp. 69-86.

[6] H. BREZIS, "Operateurs Maximaux Monotones" North Holland, Amsterdam (1973).

[7] F. BRowder and P. Hess, “Nonlinear mappings of monotone type" J. Funct. Anal. 11 (1972), pp. 251-294.

[8] A. Cellina, "On the set of solutions to Lipschitzian differential inclusions" Diff. and Integral Equations 1 (1988), pp. 495-500.

[9] G. Colombo, A. Fryszkowski, T. Rzezuchowski and V. Staicu, "Continuous selection of solution sets of Lipschitzian differential inclusions" Funkcialaj Ekvaciojto appear.

[10] N. Dunford and J. Schwartz, "Linear Operators I" Wiley, New York (1958).

[11] A. Filippov, "Classical solutions of differential equations with multivalued right hand side" SIAM J. Control Optim. 5 (1967), pp. 609-621.

[12] E. Flytzanis and N.S. Papageorgiou, "On the existence of monotone and slow solutions of differential inclusions" Math Japonica 35 (1990), pp. 399-407.

[13] A. Fryszkowski, "Continuous selections for a class of nonconvex multivalued maps" Studia Math 76 (1983), pp. 163-174.

[14] N. KenMOCHI, „Nonlinear operators of monotone type in reflexive Banach spaces and nonlinear perturbations" Hiroshima Math Jour. 4 (1974), pp. 229-263.

[15] E. Klein And A. Thompson, "Theory of Correspondences" Wiley, New York (1984). 
[16] E. NAGY, "A theorem on compact embedding for functions with values in an infinite dimensional Hilbert space" Annales Univ. Sci. Budapest, Sectio Math 29 (1986), pp. 243-245.

[17] N.S. PAPAGEORgIOU, "Convergence theorems for Banach space valued integrable multifunctions" Intern. J. Math. and Math. Sci. 10 (1987), pp. 433-442.

[18] N.S. PAPAGEORGIOU, "On measurable multifunctions with applications to random multivalued equations" Math. Japonica 32 (1987), pp. 437-464.

[19] N.S. PAPAGeorgiou, "Optimal control of nonlinear evolution inclusions" J. Optim. Theory Appl. 67 (1990), pp. 321-354.

[20] V. StaICU, "Continuous selections of solutions sets to evolution equations" SISSA 42M (1990), Trieste, Italy.

[21] D. WAGNER, "Survey of measurable selection theorems" SIAM J. Control Optim. 15 (1977), pp. 859-903.

[22] E. ZEIDLER, "Nonlinear Functional Analysis and its Applications II" Springer, New York (1990).

[23] G. Edgar, "Measurability in a Banach space Il" Indiana Univ. Math. J. 28 (1979), pp. 559-579.

National Technical University

Department of Mathematical Sciences

Zografou CAMPUS

Athens 157 73, Greece

AND

Florida Institute of TECHNOLOGY

Department of Mathematics

Melbourne, Florida 32901-6988,

U.S. A. 